\section{Nutzen der Melanomtherapie unterschätzt?}

\author{
Die traditionellen RECIST(Response Evaluation Criteria in Solid Tumors)- \\ Kriterien haben bisher gute Dienste geleistet. Ob das allerdings auch für \\ Immuntherapien gilt, war bislang nicht klar.
}

U S-Onkologen um Stephen Hodi und Jedd Wolchok vom Memorial Sloan Kettering Cancer Center in New York postulierten nach klinischen Studien mit Ipilimumab, dass auch bei Pembrolizumab die Anwendung der RECISTKriterien nicht das ganze Bild des Ansprechens liefern. In der KEYNOTE001-Studie mit 655 Melanompatienten prüften sie den Zusammenhang zwischen Gesamtüberleben und Ansprechen, beurteilt nach den RECIST-Kriterien (v1.1) bzw. den immunassoziierten Responsekriterien (irRC: immune-related response criteria, Version 2009).

Während es bei den RECIST-Kriterien z. B. als partielles Ansprechen gilt, wenn die Tumorlast um mindestens $30 \%$ im Vergleich zur Ausgangssituation abgenommen hat, verlangen die irRC-Kriterien eine Abnahme von mindestens $50 \%$. Nach RECIST werden nur maximal 5 Target-Läsionen gefordert, nach den irRC-Kriterien 15. Nicht zuletzt erfolgt die Vermessung des Tumors nach RECIST nur längs, nach irRC-Kriterien dagegen auch anhand der kurzen $\mathrm{Tu}$ mormessachse.

In der KEYNOTE-001-Studie waren 84 Patienten, die nur nach RECIST als progredient galten, nicht aber nach

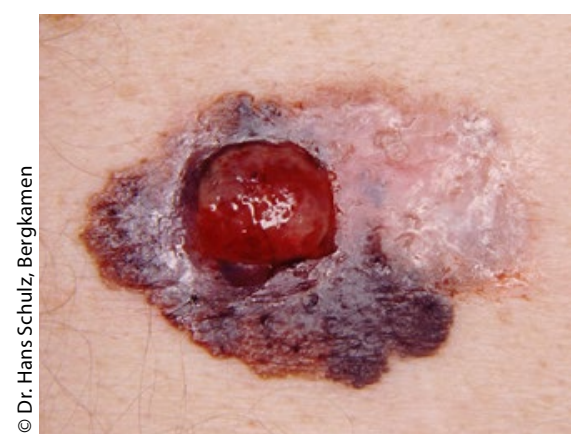

Bei der Immuntherapie des malignes Melanom sind möglicherweise andere Beurteilungskriterien als die RECIST-Kriterien relevant.
irRC-Kriterien. Bei ihnen betrug die Gesamtüberlebensrate nach zwei Jahren $37,5 \%$ und war damit höher als bei den 177 Patienten, bei denen eine Krankheitsprogression anhand beider Kriterien gleichermaßen erkannt wurde $(17,3 \%)$. Hodi und Kollegen gehen davon aus, dass in der Studie durch Verwendung der RECIST-Kriterien der Nutzen von Pembrolizumab um ungefähr $15 \%$ unterschätzt wurde. Es sei zu vermuten, dass die Patienten von der Immuntherapie auch dann noch profitierten, wenn bereits erste Zeichen einer radiografisch nachweisbaren Progression erkennbar seien, so die Ärzte. Die irRC-Kriterien könnten Therapeuten helfen, die Behandlung nicht vorzeitig abzubrechen.

Fazit: In der retrospektiven Studie mit Melanompatienten wurde das Ansprechen unter Verwendung der traditionellen RECIST-Kriterien unterschätzt. Die Verwendung immunspezifischer Kriterien könnte das vielleicht künftig verhindern. Zuvor sind jedoch noch prospektive Studien erforderlich. Peter Leiner

Hodi FS et al. Evaluation of Immune-Related Response Criteria and RECIST v1.1 in Patients With Advanced Melanoma Treated With Pembrolizumab. J Clin Oncol. 2016;34(13):1510-7.

nostischen Nutzen hat. Allenfalls zeigte sich hier ein Vorteil der kompletten Lymphknotendissektion gegenüber abwartendem Beobachten mit Blick auf das krankheitsfreie Überleben. Obwohl signifikant (Hazard Ratio 0,58; p = $0,028)$, schlug sich dieser Effekt nicht in einem besseren Gesamtüberleben nieder.

Fazit: Die hochdosierte IFN-Gabe verbesserte weder krankheitsspezifisches noch Gesamtüberleben, und bei negativer Histologie und Immunchemie wirkte sich eine positive RT-PCR auf melanomspezifische RNA nicht auf das Gesamtüberleben aus. Robert Bublak

McMasters KM et al. Final Results of the Sunbelt Melanoma Trial: A Multi-Institutional Prospective Randomized Phase III Study Evaluating the Role of Adjuvant High-Dose Interferon Alfa- $2 \mathrm{~b}$ and Completion Lymph Node Dissection for Patients Staged by Sentinel Lymph Node Biopsy. J Clin Oncol. 2016;34(10):1079-86. 